УДК 664.723 .047

\title{
ЦИКЛИЧЕСКАЯ МИКРОВОЛНОВАЯ СУШКА ПЛОТНОГО СЛОЯ ЗЕРНОВЫХ МАТЕРИАЛОВ
}

Бошкова И.Л., докт. техн. наук, Волгушева Н.В., канд. техн. наук

Одесская наџиональная академия пищевых технологий. Дворянская 1/3, Одесса 65012, Украина

Наведено результати експериментального дослідження кінетики циклічної мікрохвильової сушки щільного шару зерна при різних тривалостях продувки. Визначено вплив температури повітря при продуванні шару на основні характеристики процесу. Визначені режимні параметри для оптимальної циклічної сушки, встановлена якісна залежність швидкості сушіння в різні періоди циклів від тривалості процесу.

Библ. 6, табл. 3, рис. 3.
Приведены результаты экспериментального исследования кинетики циклической микроволновой сушки плотного слоя зерна при различных длительностях продувки. Установлено влияние температуры воздуха при продувке слоя на основные характеристики процесса. Определены режимные параметры для оптимальной циклической сушки, установлена качественная зависимость скорости сушки в различные периоды циклов от длительности процесса.
The results of experimental studies of the kinetics of cyclic microwave drying for thick layer of grain at different durations purge are presented. The effect of temperature of air while purging layer on the main characteristics of the process established. Operating parameters for optimum cyclic drying identified, qualitative dependence of the drying rate at different periods of cycles of processing time established.

Ключевые слова: сушка, зерно, плотный слой, эффективность, микроволновой подвод энергии.

$N$ - скорость сушки;

$Q$ - затраты энергии;

$t$ - температура;

$U$ - влагосодержание;

$\tau$ - время.

Индексы нижние:

Сушка зерновых требует больших затрат энергии, для снижения которых совершенствуются существующие и разрабатываются новые инновационные технологии. Как показывают результаты исследований, в условиях микроволнового нагрева возникает возможность сокращения удельных затрат энергии [1-3], при этом комбинированные способы позволяют поддерживать требуемый температурный режим и высокую скорость сушки [4]. Одним из таких комбинированных методов является циклическая сушка, при которой периоды подвода микроволновой энергии чередуются с периодами продувки слоя сушильным агентом (к примеру, воздухом). В то же время для получения рациональных ре-

$$
\begin{aligned}
& \text { в - воздух; } \\
& \text { к- конечное; } \\
& M B \text { - микроволновая; } \\
& \text { прод - продувка; } \\
& \text { ср - среднее; } \\
& \text { уд - удельные. }
\end{aligned}
$$

жимов целесообразно провести анализ не только средних величин, характеризующих интенсивность сушки за весь период, а проследить их изменение в каждом цикле.

Целью исследований являлось определение влияния продолжительности продувки слоя зерна воздухом, без предварительного подогрева и нагретым, на основные характеристики процесса сушки (средняя температура материала, скорость сушки, удельные энергозатраты), и выбор оптимального режима при циклическом подводе микроволновой энергии. Схема микроволновой экспериментальной установки для исследования процессов тепломассопереноса при сушке зерновых материалов представлена на рис. 1. 


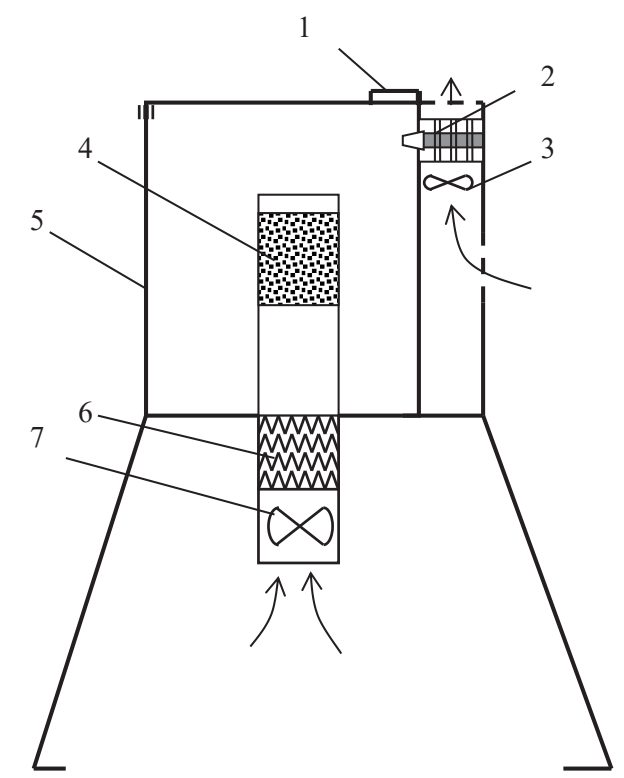

Рис. 1. Схема установки для исследования кинетики сушки зерновых материалов при микроволновом и конвективном нагреве:

1 - дверца, 2 -магнетрон, 3 - вентилятор системы охлаждения магнетрона, 4 - экспериментальная ячейка с материалом для исследований, 5 - рабочая камера, 6 - электронагреватель, 7 - вентилятор.

Установка обеспечивает проведение исследований при микроволновой, микроволновоконвективной и конвективной сушке. Внутри рабочей камеры установлен воздуховод из радиопрозрачного материала, в который помещалась ячейка, изготовленная в форме параллелепипеда из сетчатого материала. В экспериментальную ячейку засыпалось заданное количество зерна. Методика проведения экспериментов состояла в следующем. В микроволновую установку загружалось 100 г зерна (овес) с начальным влагосодержанием 0,2 кг/кг. Сушка зерна проходила в циклическом режиме. Длительность периода MB нагрева во всех опытах составляла $\tau=10 \mathrm{c}$, выходная мощность магнетрона соответствовала 600 Вт. Длительность периода продувки варьировалась: 10, 20 и 30 с. После каждого из периодов образец извлекался, проводились замеры температуры и массы зерна, после чего в ячейку засыпалась новая порция зерна с таким же начальным влагосодержанием, и процесс длился на соответствующий период больше. Температура воздуха составляла $20^{\circ} \mathrm{C}$, скорость фильтрации в слое зерна - 1 м/c, расход - 0,0118 кг/с. Начальная температура зерна $-20{ }^{\circ} \mathrm{C}$. Результаты обработки экспериментальных данных, полученных при продувке слоя ненагретым воздухом (конечные для каждого из периодов влагосодержания $u_{k}$, температура $t_{c p}$ и скорость сушки $N$ ) приведены в табл. 1.

Увеличение длительности продувки приводит к снижению температуры слоя, при этом влагосодержание в конце всех трех опытов практически одинаково.

Исследование сушки зерна при продувке предварительно нагретым воздухом проводилось по приведенной выше методике, воздух нагревался электронагревателем 6 (рис. 1). Температура зерна в период продувки нагретым воздухом снижается менее существенно, чем при продувке холодным воздухом (табл. 2), что определяет большую равномерность сушки. Скорость воздуха $-1 \mathrm{M} / \mathrm{c}$.

Анализируя данные по сушке с применением нагретого воздуха, можно отметить, что средняя скорость в период продувки длительностью $\tau_{\beta}=10$ с выше, а удельные затраты ниже, чем при $\tau_{b}^{b}=20 \mathrm{c}$, поэтому этот режим является предпочтительным. Однако продувка нагретым воздухом, даже при температуре $50{ }^{\circ} \mathrm{C}$, приводила к недопустимому росту температуры зерна. Сравнительный анализ результатов позволяет сделать вывод, что оптимальной является сушка с продувкой слоя без предварительного подогрева воздуха. В этих режимах при высокой скорости сушки, сопоставимой со скоростью при продувке нагретым воздухом, удельные затраты энергии ниже. Скорость сушки при длительности продувки 10 с выше для воздуха при $t=20^{\circ} \mathrm{C}$, чем при $t_{b}=50{ }^{\circ} \mathrm{C}$, Для режимов с $t_{6}=20^{\circ} \mathrm{C}$ при продувке слоя ненагретым воздухом скорость сушки резко снижается, что связано с понижением температуры зерна. На интенсивность выхода влаги из зерновки оказывает влияние возникновение избыточного давления с ростом температуры при микроволновом нагреве. В соответствии с данными [5], при сушке в электромагнитном поле при температуре от $45{ }^{\circ} \mathrm{C}$ до $95^{\circ} \mathrm{C}$ (а по результатам наших исследований микроволновой сушки плотного слоя зерна - для $70{ }^{\circ} \mathrm{C}$ [4]), возникает градиент давления, который интенсифицирует 
перенос влаги. Отмечается, что при выключении источника электромагнитного поля избыточное давление релаксирует не мгновенно вследствие сопротивления молярному переносу внутри тела. Если до температуры $70{ }^{\circ} \mathrm{C}$ избыточное давление равно нулю, и перенос молекул воды описывается законами неизотермической диффузии влаги [5], то при дальнейшем повышении температуры возникающий градиент давления приводит к интенсификации выхода влаги к поверхности зерновки.

Табл. 1. Характеристики сушки плотного слоя зерна (овес) при циклическом подводе микро-волновой энергии. Продувка слоя воздухом без предварительного подогрева

\begin{tabular}{|c|c|c|c|c|c|c|c|c|c|c|}
\hline Цикл & Период & $\begin{array}{c}u_{\kappa^{\prime}} \\
\kappa \Gamma / \kappa \Gamma\end{array}$ & $\begin{array}{l}t_{c p}, \\
{ }^{\circ} \mathrm{C}\end{array}$ & $\begin{array}{l}, \\
\mathrm{c}^{-1} \\
\end{array}$ & $\begin{array}{c}u_{k^{\prime}} \\
\kappa \Gamma / \kappa \Gamma \\
\end{array}$ & $\begin{array}{l}t_{c p}, \\
{ }^{\circ} \mathrm{C}\end{array}$ & $\begin{array}{l}N, \\
\mathrm{c}^{-1} \\
\end{array}$ & $\begin{array}{c}u_{k^{\prime}} \\
\kappa \Gamma / \kappa \Gamma \\
\end{array}$ & $\begin{array}{l}t_{c p}, \\
{ }^{\circ} \mathrm{C}\end{array}$ & $\begin{array}{l}N, \\
\mathrm{c}^{-1} \\
\end{array}$ \\
\hline & & \multicolumn{3}{|c|}{$\tau_{\text {mр0 }}=10 \mathrm{c}$} & \multicolumn{3}{|c|}{$\tau_{\text {прол }}=20 \mathrm{c}$} & \multicolumn{3}{|c|}{$\tau_{\text {口рол }}=30 \mathrm{c}$} \\
\hline \multirow{2}{*}{1} & $\mathrm{MB}$ & 0,199 & 53,5 & 0,0001 & 0,1990 & 41,5 & 0,0001 & 0,198 & 40,3 & 0,0002 \\
\hline & $\Pi$ & 0,195 & 46,5 & 0,0004 & 0,1930 & 33 & 0,0003 & 0,192 & 27 & 0,0002 \\
\hline \multirow{2}{*}{2} & $\mathrm{MB}$ & 0,194 & 70,5 & 0,0001 & 0,1913 & 51,5 & 0,00017 & 0,191 & 44 & 0,0001 \\
\hline & $\Pi$ & 0,190 & 57 & 0,0004 & 0,1827 & 45,5 & 0,00043 & 0,184 & 39,3 & 0,0002 \\
\hline \multirow{2}{*}{3} & $\mathrm{MB}$ & 0,187 & 74 & 0,0003 & 0,1822 & 59,5 & 0,00005 & 0,183 & 52,7 & 0,0001 \\
\hline & $\Pi$ & 0,179 & 61,5 & 0,0008 & 0,1748 & 44 & 0,00037 & 0,173 & 44 & 0,0003 \\
\hline \multirow{2}{*}{4} & $\mathrm{MB}$ & 0,176 & 81,5 & 0,0003 & 0,1685 & 68,5 & 0,00017 & 0,172 & 58 & 0,0001 \\
\hline & $\Pi$ & 0,171 & 71,5 & 0,0005 & 0,1649 & 49,5 & 0,00018 & 0,168 & 42,7 & 0,0001 \\
\hline \multirow{2}{*}{5} & $\mathrm{MB}$ & 0,165 & 71 & 0,0006 & 0,1642 & 72,5 & 0,00007 & 0,165 & 65,7 & 0,0003 \\
\hline & $\Pi$ & 0,161 & 66,5 & 0,0004 & 0,1608 & 61 & 0,00017 & 0,157 & 51 & 0,0003 \\
\hline \multirow[t]{2}{*}{6} & $\mathrm{MB}$ & 0,154 & 81,5 & 0,0007 & 0,1547 & 63 & 0,00061 & 0,155 & 57,7 & 0,0002 \\
\hline & $\Pi$ & 0,15 & 69,5 & 0,0004 & 0,1504 & 54,5 & 0,00022 & 0,145 & 61 & 0,0003 \\
\hline 7 & $\mathrm{MB}$ & 0,144 & 80,5 & 0,0006 & 0,1480 & 68 & 0,00024 & 0.144 & 63,3 & 0,0001 \\
\hline
\end{tabular}

Табл. 2. Характеристики сушки плотного слоя зерна (овес) при циклическом подводе микроволновой энергии. Продувка слоя воздухом при температуре на входе $t_{\theta}=50{ }^{\circ} \mathrm{C}$

\begin{tabular}{|c|c|c|c|c|c|c|c|}
\hline Цикл & Период & $\begin{array}{c}\mathrm{u}_{\mathrm{k}}, \\
\mathrm{\kappa} \Gamma / \mathrm{\kappa} \Gamma\end{array}$ & $\begin{array}{l}\mathrm{t}_{\mathrm{cp}}, \\
{ }^{\circ} \mathrm{C}\end{array}$ & $\begin{array}{l}\mathrm{N}, \\
\mathrm{c}^{-1}\end{array}$ & $\begin{array}{c}\mathrm{u}_{\mathrm{k}} \\
\mathrm{\kappa} / \mathrm{K} \Gamma\end{array}$ & $\begin{array}{l}\mathrm{t}_{\mathrm{cp}}, \\
{ }^{\mathrm{o}} \mathrm{C}\end{array}$ & $\begin{array}{l}\mathrm{N}, \\
\mathrm{c}^{-1}\end{array}$ \\
\hline \multirow{2}{*}{1} & $\mathrm{MB}$ & 0,199 & 46 & 0,0001 & 0,199 & 46,00 & 0,0001 \\
\hline & $\Pi$ & 0,196 & 36,33 & 0,0003 & 0,194 & 32,33 & 0,00025 \\
\hline \multirow{2}{*}{2} & $\mathrm{MB}$ & 0,195 & 54,33 & 0,0001 & 0,193 & 50,67 & 0,0001 \\
\hline & $\Pi$ & 0,189 & 47 & 0,0006 & 0,185 & 51,67 & 0,0004 \\
\hline \multirow{2}{*}{3} & $\mathrm{MB}$ & 0,188 & 68,33 & 0,0001 & 0,1845 & 65,00 & 0,00005 \\
\hline & $\Pi$ & 0,182 & 67 & 0,0006 & 0,174 & 46,00 & 0,00053 \\
\hline \multirow{2}{*}{4} & $\mathrm{MB}$ & 0,178 & 72,67 & 0,0004 & 0,1734 & 64,33 & 0,00006 \\
\hline & $\Pi$ & 0,174 & 70,33 & 0,0004 & 0,165 & 64,00 & 0,00042 \\
\hline \multirow{2}{*}{5} & $\mathrm{MB}$ & 0,171 & 83,67 & 0,0003 & 0,1646 & 70,67 & 0,00004 \\
\hline & $\Pi$ & 0,163 & 76 & 0,0008 & 0,155 & 64,33 & 0,00048 \\
\hline \multirow{2}{*}{6} & $\mathrm{MB}$ & 0,158 & 86,33 & 0,0005 & 0,1536 & 86,00 & 0,00014 \\
\hline & $\Pi$ & 0,153 & 64 & 0,0005 & 0,1435 & 74,33 & 0,00051 \\
\hline 7 & $\mathrm{MB}$ & 0,150 & 95,33 & 0,0003 & 0,1397 & 91,00 & 0,00038 \\
\hline
\end{tabular}


Анализ полученных результатов позволяет предположить, что физическая картина циклической сушки состоит в следующем. После снятия МВ нагрузки и продувки слоя уносится пар из межзернового пространства, а подступившая к поверхности влага частично - испаряется и частично - вновь сорбируется зерновкой. Таким образом, длительность продувки должна быть такой, чтобы вся испарившаяся влага удалялась из межзернового пространства, а релаксационные процессы в зерновках не завершились, что в следующем цикле дает возможность не затрачивать энергию на повторный перенос влаги к поверхности зернови. Это объясняет экспериментальный результат, полученный в данных исследованиях, согласно которому оптимальному режиму циклической сушки соответствует минимальная длительность продувки ненагретым воздухом.
Для оценки общей энергетической эффективности циклической сушки выполнены расчеты средних за весь процесс значений удельных затрат энергии $Q_{v d}$, скорости сушки $N_{c p}$, и сред-

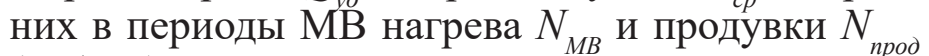
(табл. 3). Удельные затраты энергии существенно ниже в режимах, применяющих воздух без подогрева. Следует отметить, что значения удельных затрат определяются конструкцией экспериментальной установки, которая не являлась оптимальной по использованию микроволновой энергии, излучаемой магнетроном. Однако полученные данные позволяют проводить сравнительную оценку и определять режимные параметры, при которых наблюдается снижение затрат энергии. Анализ таблицы показывает, что для всех исследованных режимов средняя за процесс скорость сушки выше, чем в применяемых конвективных сушилках [6].

Табл. 3. Сравнительные характеристики циклической сушки при различных входных температурах воздуха и длительности продувки

\begin{tabular}{|c|c|c|c|c|c|}
\hline$t^{\circ}{ }^{\circ} \mathrm{C}$ & \multicolumn{3}{|c|}{20} & \multicolumn{2}{|c|}{50} \\
\hline$\tau_{\text {mpor }},{ }^{\circ} \mathrm{C}$ & 10 & 20 & 30 & 10 & 20 \\
\hline $\begin{array}{l}\text { Удельные затраты энергии } \\
Q_{v \vartheta}, \text { МДж/кг }\end{array}$ & 9,07 & 9,68 & 8,96 & 11,72 & 14,33 \\
\hline $\begin{array}{l}\text { Средняя скорость сушки } \\
N_{c}, \mathrm{c}^{-1}\end{array}$ & 0,00043 & 0,000274 & 0,000224 & 0,00038 & 0,000316 \\
\hline $\begin{array}{l}\text { Средняя скорость МВ сушки } \\
N_{M B}, \mathrm{c}^{-1}\end{array}$ & 0,000343 & 0,000201 & 0,000157 & 0,00025 & 0,000124 \\
\hline $\begin{array}{l}\text { Средняя скорость за периоды } \\
\text { продувки } \\
N_{n p o,} \mathrm{c}^{-1}\end{array}$ & 0,000483 & 0,000278 & 0,000233 & 0,000533 & 0,000432 \\
\hline
\end{tabular}

На рис. 2 приведены результаты расчетов скорости сушки для различных периодов. Анализ данных приводит к выводу, что при продувке как нагретым, так и ненагретым воздухом, вначале скорость сушки выше в периоды продувки, а в конце - значительно усиливается скорость сушки в периоды МВ нагрева, причем для ненагретого воздуха скорость МВ сушки становится больше, чем в периоды продувки. При использовании нагретого воздуха температура материала в конце эксперимента была выше на $10 \ldots 20{ }^{\circ} \mathrm{C}$ по сравнению с ненагретым. Результаты экспе- риментов показывают, что на значение скорости сушки в периоды МВ нагрева и в периоды продувки оказывает влияние температура материала и его влагосодержание. С увеличением $\tau_{\text {прод }}$ от 10 с до 20 с (рис. 2) скорость сушки снижается для всех периодов, что говорит об общей нерациональности увеличения длительности продувки. При времени 80 с (рис. 2, а) и 150 с (рис. 2, б), что соответствует четвертому циклу для $\tau_{\text {прод }}=10$ с и пятому для $\tau_{\text {прод }}=20$ с, и далее, отмечается существенное превышение скорости MB сушки в сравнении со скоростью в перио- 
ды продувки. При этом, как видно из табл. 1, для данных, полученных при $\tau_{\text {прод }}=20 \mathrm{c}$, этот цикл характеризуется более низкими значениями температур и влагосодержания по сравнению с данными, полученными при $\tau_{\text {прод }}=10 \mathrm{c}$.

В период продувки нагретым воздухом скорость сушки всегда была выше (рис. 3), однако после четвертого цикла для $\tau_{\text {прод }}=10$ с и пятого для $\tau_{\text {прод }}=20$ с начинает увеличиваться вклад МВ сушки. Как и при продувке воздухом без подогрева, при $\tau_{\text {прод }}=20$ с граничный цикл характеризуется более низкими значениями температур и влагосодержания по сравнению с данными, полученными при $\tau_{\text {прод }}=10$ с (табл. 2).
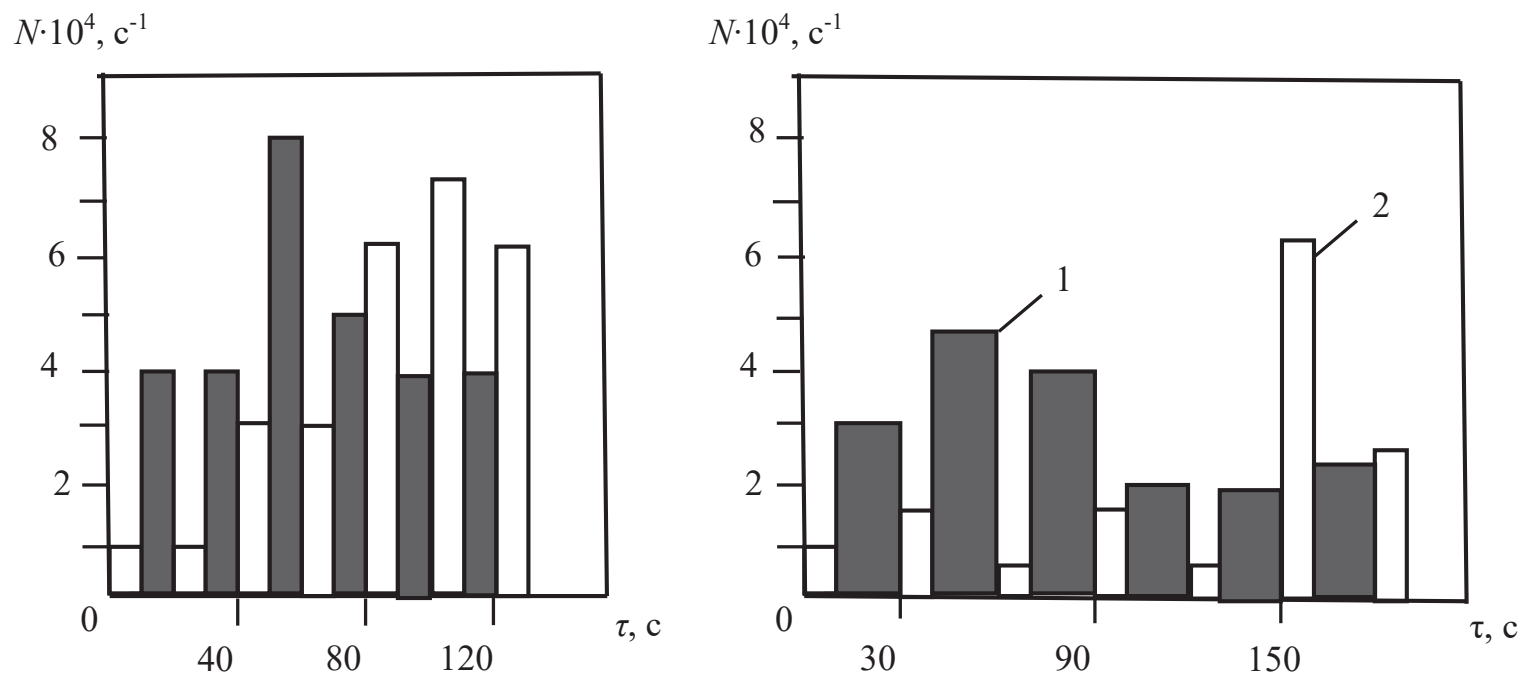

Рис. 2. Зависимость скорости циклической суики плотного слоя зерна от времени при продувке воздухом без предварительного подогрева:

1 - период продувки, 2 - период МВ нагрева. Длительность МВ нагрева -10 с. $\boldsymbol{a}-\tau_{\text {прод }}=10 \mathrm{c}, \boldsymbol{\sigma}-\tau_{\text {прод }}=20 \mathrm{c}$.

$N \cdot 10^{4}, \mathrm{c}^{-1}$

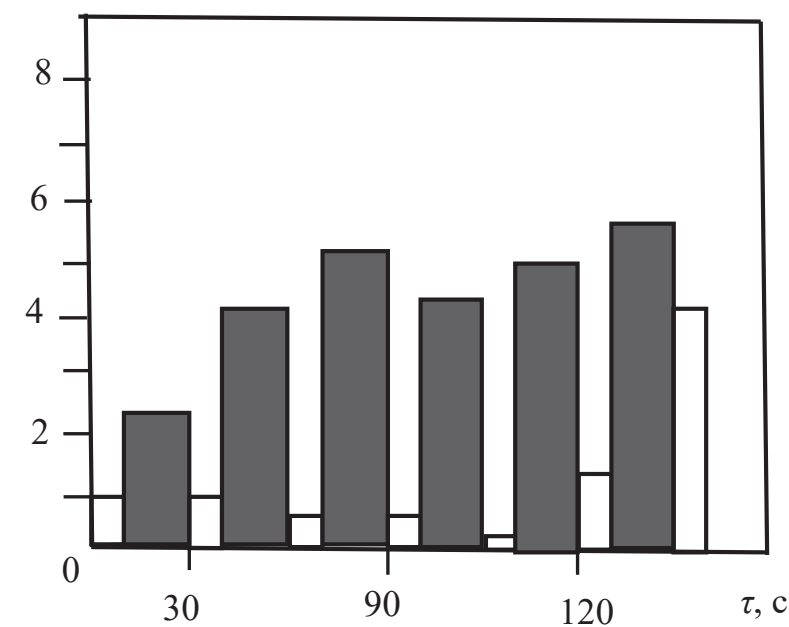

$N \cdot 10^{4}, \mathrm{c}^{-1}$

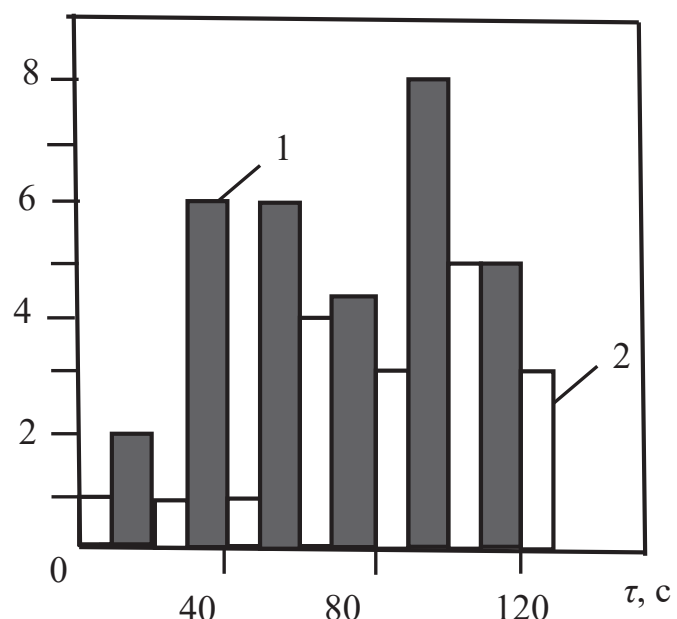

Рис. 3. Зависимость скорости циклической суики плотного слоя зерна от времени при продувке воздухом с температурой на входе $50{ }^{\circ} \mathrm{C}$ :

1 - период продувки, 2 - период МВ нагрева. Длительность МВ нагрева - 10 с. $\boldsymbol{a}-\tau_{\text {прод }}=10 \mathrm{c}, \boldsymbol{\sigma}-\tau_{\text {прод }}=20 \mathrm{c}$. 
Количественной зависимости скорости циклической сушки от значений влагосодержания и температуры слоя на данном этапе определить не удалось. Тем не менее, полученные данные свидетельствуют о существенном перераспределении вклада продувки и МВ нагрева в процессе сушки и позволяют предположить, что существует возможность управления путем изменения соотношения периодов продувка-МВ нагрев.

Получено, что варьированием длительностью периодов МВ нагрева и продувки достигается возможность установления интенсивного и энергоэффективного режима сушки. Для получения более полной информации о рациональных режимах сушки, использующей микроволновой нагрев, целесообразно установить изменение основных характеристик процесса по времени для одновременной микроволново-конвекетивной сушки и провести сопоставительный анализ результатов.

\section{Выводы}

1. Оптимальному режиму циклической микроволновой сушки соответствует минимальная из ряда исследованных длительность продувки слоя зерна (10 с) воздухом без его предварительного подогрева.

2. Скорость сушки в первой части процесса всегда выше в периоды продувки, во второй части значительно усиливается скорость в период MB нагрева, причем для воздуха без предварительного подогрева скорость МВ сушки становится выше, чем в периоды продувки. На значение скорости сушки в периоды МВ нагрева и в периоды продувки оказывает влияние температура материала и его влагосодержание.

3. Температура слоя зерна при микроволновом нагреве является главным определяющим фактором, влияющим на скорость сушки. Варьированием длительностью периодов МВ нагрева и продувки достигается возможность установления интенсивного и энергоэффективного режима сушки. Представляется целесообразным установить экспериментальным путем изменение основных характеристик процесса по времени для одновременной микроволново-конвективной сушки и провести сопоставительный анализ результатов.

\section{ЛИТЕРАТУРА}

1. Advances in Bioprocessing Engineering / Editors H. Yang, J. Tang. London. Advances in Agricultural Science and Technology. Vol. 1. Editors H. Yang, J. Tang. London: World Scientific, 2002. $172 \mathrm{p}$.

2. Календерьян В. А., Бошкова И.Л., Волгушева Н.B. Кинетика микроволновой сушки сыпучего органического материала // ИФЖ, 2006 .- №3, Т.79. - С. 123-127.

3. Бошкова И.Л., Волгушева Н.В., Дементьева Т.Ю. Развитие энергосберегающей сушилки для зерна на основе микроволнового подогрева // ТЭПРБП: Южно-Уральский гос. ун-т. - Челябинск, 2013. - С. 365-368.

4. Волгушева Н.В. Кінетика сушіння щільного шару дисперсного матеріалу (на прикладі гречки) при різних способах підведення теплоти / Автореф. дис. канд. техн. наук. /Одеса: ОДАХ, 2005. - $18 \mathrm{c}$.

5. Лыков A.B. Теория сушки. - М.: Энергия, 1968. $-472 \mathrm{c}$.

6. Дементьєва Т. Ю. Інтенсифікація процесів тепловологопереносу при сушінні зернового матеріалу з застосуванням мікрохвильового електромагнітного поля / Автореф. дис. канд. техн. наук /Одеса: ОДАХ, 2012. - 22 с. 


\section{CYCLIC MICROWAVE DRYING DENSE LAYER OF GRAIN MATERIALS}

\section{Boshkova I.L., Volgusheva N.V.}

Odessa national academy of food technologies, Dvoryanskaya 1/5, Odessa 65082, Ukraine

The results of experimental studies of drying dense layer grain under the cyclical supply to microwave energy for different exposition and at different inlet air temperature are presented. It has found that drying without preheating is optimal. A physical picture cyclic drying, which explains the experimental result, according to which the optimal regime corresponds to the minimum duration of the purge air out of the test series, is formulated. It has shown that the drying rate significantly redistribute between periods during the whole process, and speed microwave drying becomes higher in the final stage than in the purge period for the air without preheating.

References 6, tables 3, figures 3.

Key words: drying, grain, dense layer, efficiency, a microwave energy supply
1. Advances in Bioprocessing Engineering / Editors H. Yang, J. Tang. London. Advances in Agricultural Science and Technology. Vol. 1. Editors H. Yang, J. Tang. London: World Scientific, 2002. 172 p.

2. Kalenderyan V.A., Boshkova I.L., Volgusheva N.V. Kinetics of Microwave Drying of a Free-Flowing Organic Material // IFZh, 2006 - P. 123-127 (Rus).

3. Boshkova I.L., Volgusheva N.V., Dementeva T.Yu. The development of energy-saving grain dryers based on microwave heating // TEPRBP: YuzhnoUralskiy gos. un-t. - Chelyabinsk, 2013 - P. 368-368 (Rus).

4. Volgusheva N.V. Kinetics drying dense bed of particulate material (for example buckwheat ) for various methods for supplying heat / Avtoref. dis. kand. tehn. nauk. /Odesa: ODAH, 2005. 18p. (Ukr).

5. Lyikov A.V. Theory of Drying. - M.: Energiya, 1968. - 472p. (Rus).

6. Dementeva T. Yu. Intensification of processes of heat and moisture transfer in drying grain material under microwave electromagnetic field / Avtoref. dis. kand. tehn. nauk /Odesa: ODAH, 2012. -22 p. (Ukr). 